\title{
Computer Assisted Diagnosis of Upper Limbs Using Markerless Mocap and Discriminant Bands of Normal Movement
}

\author{
V. Javier Romano M., Manuel Martin O., and Arturo Olvera L. \\ Faculty of Computer Science \\ Autonomous University of Puebla \\ Av. San Claudio y 14 sur, C.P. 72570, Puebla Pue., Mexico \\ \{victor.romano, mmartin, aolvera\}@cs.buap. $\mathrm{mx}$
}

\begin{abstract}
Computer vision has become and important tool in several fields of science, like Medicine and Biomechanics. In this work we made an analysis of upper limb movemnts from a kinematic strategy. The information is captured through Markerless Mocap, we obtain the spatial position of the most important joints of the body. We take this numeric values to process and translate them into specific information for the specialist in the physiotherapy field. As a complementary task we create discriminant bands of normal human movement using several curves of behaviour of the local population in an specifc group with similar physical characteristics. An specific support to the diagnosis is the quatitative data obtained from the curve of movement in a standard motion of a patient and the results of the comparisons with the discriminant bands. The system is programmed using Opensource tools with an easy installation without demanding special physical conditions and as a not expensive approach.
\end{abstract}

Key words: Computer vision, markerless MoCap, computer assisted diagnosis.

\section{Introduction}

Movement is one of the vital tasks of human lifestyle, it help us in our working activities, walking, lifting objects, practicing sports and even eating or doing recreational activities. Most of this movement involves the torso and the extremities, these parts of the body are vulnerable of different factors including diseases that can alter joints, muscles, bones or nerves that are involved in some movements, another factor are injuries limiting the range of motion and the third factor is an unavoidable problem every human has: Age, as we grow older the muscles loose their flexibility, the joints their elasticity and we are more exposed to external pathogens factors. As the average of life span is growing bigger we need more specialized tools in detection and treatment of patients with motion problems [1]. 
Computer vision is growing fastly because of the great improvements in proccesing speeds, increased amount of storage and the increasing level of detail that videocameras now have. We introduce a system that helps physiotherapists when a diagnosis is made as a great potential tool, with a non-expensive cost and easy usage, only using a optical capture device, the tool offers a non-invasive Markerless Mocap that has several advantages because is not as demanding as wearing exoskeletons like the ones used by Xsens [2] or the vest proposed by the Ergonomics' division of the Zaragoza University [6]. Using a capture and a processing subsystem we obtain numerical data about the position of the main body joints through a basic movement routine that streams the depth and RGB video, then with this information a Physiotherapist can use a Results subsystem to compare the motion of a patient with the normal preestimated movement of a certain local population and also can be compared with its own motions of previous medical checkups; the purpose of this is to evaluate the progress of a rehabilitacional therapy, to reinforce a diagnosis of improvement or deterioration of a patient's motion made by the specialist, to route the diagnosis on the path of an specific pathology. The assisted diagnosis is more complete because it includes the experience, intuition and knowledge of the specialist and it is based on numerical values, graphics and videos provided and stored in a computer.

\section{Methodology}

The target of the present project is to help on diagnosis tasks and tracking of rehabilitational therapies, so it should offer simple and organized interfaces. It offers the capture of movement in a kinematic approach of upper limbs, it registers the behavior through time, the diagnosis for each appointment and graphical results that help to interpret the numerical data. Also it stores the depth streams and the three-channels (RGB) video and links them with the medical history to use them at the study of a rehab progress, in a general way the available information, processed, visualized and interpreted helps a better medical practice. This work helps in the inspection of active, passive movements and movements against resistance that are common stages of a kinesio therapy appointment; the most conclusive data obtained using this proccess are the recognition of motion range limitations and the recognition of movement stages with the presence of pain.

The study is targeted to the five basic moves of the shoulder joint that are Abduction-Adduction, Flection- Extension, Hyperextension, Internal-External Rotation and Horizontal Abduction- Adduction, we selected the shoulder because it is the most affected upper body joint in the specialized clinic in physical rehabilitation of the physiotheraphy school in Autonomous University of Puebla. Focusing to the shoulder joint, if we get a limitation of the motion range it could be related to one of these pathologies: Reflex sympathetic dystrophy, recurrent dislocation, subluxations, Periarthritis scapular - humeral ankylosing. If the patient presents pain in a specific range of angles could be related with: Brachial plexus syndrome, Narrowing syndrome (Pain from 60 to 120 on Abduction), 
Impingment syndrome (Pain from 80 to 120 on Abduction), Anterior cruciate ligament injury or hemiplejia among many other diseases that could be detected visually [7]. Regarding the capture system we use an optical method that allows depth streams over an electromagnetic, mechanical or an ultrasonic device because it satisfies the accuracy requirements when measuring the position of joints, its approachability, it is easy to install and set up. We are using Markerless mocap over a skeleton model so we can proccess the information in real time.

To develop the system we used the unified proccess model that allow us to divide the entire process in different stages so we can detect errors at early stages creating in an iterative way a more sturdy solution. The analysis of requirements, the first stage, was based on contributions of an expert of physiotheraphy to obtain the medical background, the technical part was stablished by the working group.

\section{System description}

Motion Capture (Mocap) is the process to capture the events of real movement, measures the change of position, the angle of an object in a scene to traduce it on usable mathematics values obtained from the tracking of marks through time [8] [9]. We use optical Mocap so the adquisition of information is only visual, the system uses a depth sensor to capture the information according to the Markerless Mocap type, with this approach we do not require external elements attached to a person or to his or her clothes, this is the most important advantage of markerless mocap because it allows the captured subject to wear its own clothes and without a specific suit or gear, it also helps to move as "natural" as possible. To avoid the auto-oclusion of parts of the body, one of the biggest problems with markerless, we use a starting position in a frontal angle with the hands separated from the rest of the body (Figure 1) and during the lenght of the motion routine there should not be any external obstruction.

Another advantage of Markerless Mocap with optical devices is the close relationship with the Kinematic approach, so we study the movement only as a change of position or angle of any joint without taking into consideration the forces that provoke it [5]. The system is divided into three subsystems, the first

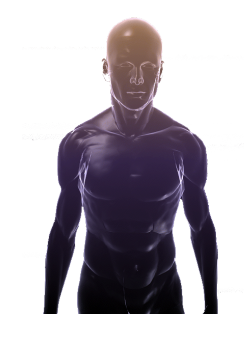

Fig. 1. Initial Position to avoid the auto-oclusion 
one is Capture subsystem that was implemented with Visual $\mathrm{C}++$ and uses the Openni Library [3] which contains many programmed routines including calibration and basic use of cameras and depth sensors, additionally we use Nite middleware [4] that help us to obtain the tracking of a person and the numerical values of specific joints through time, in addition we use OpenGL and Glui for the visual elements of the interface. The joints that we use are: Head, Neck, Chest, Left-Right Shoulder, Left-Right Elbow, Left-Righ Hand and Left-Right edge of the Hip. Connecting each pair of adjacent point we create a line, the set of lines creates a basic skeleton that represents the captured subject, this skeleton-like structure has low cost -computational talking- so it shows the results in real-time.

We chose the Microsoft Kinect Sensor as the main capture device, it allows to store the color stream and depth stream separately and with its 30 Frames Per Second of capture rate, we can obtain the whole motion curve without losing any information. As an additional task of this subsystem we also use a PS3 Eye Camera to show in a television, a mirror-like video for the patient to look himself and an animation of what curve of motion he has to follow. To use this, the patient has to stand in the front of the cameras in the initial position facing them, in Figure 2 we see the assembly of the capture subsystem.

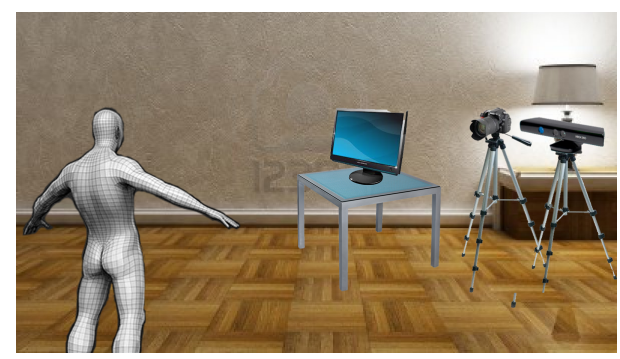

Fig. 2. Capture Subsystem Assembly

The second subsystem is the Processing one, here we take the files obtained from the capture, that have the spatial positions of each of the joints mentioned before, to create curves of motion that define the movement through time and we process them to get the final result. The first thing of the process, is reducing the noise that the sensor or any exterior factor had created, to do that we apply the rolling average and the simple exponential smoothing.

$$
\text { RollingAverage }=\frac{P_{M}+P_{M-1} \ldots+P_{M-(n-1)}}{n} .
$$

Where each $P i$ represents a joint position in a i-time and $P i+1$ the same point in the next time instant, $n$ represents the amount of points selected to create the average. The second step is to decide how many humps does the curve has, 
according to the movement and the selected axis, this is shown in Table 1.

\begin{tabular}{|l|c|c|c|}
\hline Movement & \multicolumn{1}{|c|}{ X } & Y & Z \\
\hline Abduction & 2 Humps & 1 Hump & No Hump \\
Horizontal Adduction & 1 Hump & No Hump & 2 Humps \\
Flection & No Hump & 1 Hump & 2 Humps \\
Hyperextension & No Hump & 1 Hump & 1 Hump \\
Rotation & No Hump & 1 Hump & 2 Humps \\
\hline
\end{tabular}

Table 1. Type of movement, depending on the amount of humps

The third step is removing the static parts, the time lapses where there is no change, usually this happens at the starting point or the end of each curve, these timeslots are not important to this work. They are cleaned selecting the point near the maximum and mininum range. The next step is create discriminant bands of normal movement, to do that we need to normalize curves regarding amplitude and time, this normalization needs to be done because the physical differences and times of movement of each subject. The normalization is done using the inverse linear interpolation for each separated half to correct the asimmetry of different upward and downward phases.

$$
f_{1}(x)=f\left(x_{0}\right)+\frac{f\left(x_{1}\right)-f\left(x_{0}\right)}{x_{1}-x_{0}}\left(x-x_{0}\right) .
$$

Where $X i$ represents the two possible spatial positions in which $X$ can be located and $f(x)$ is to evaluate a point in a line equation. To create the discriminant bands we calculate the arithmetic mean, then we add the average standard deviation and substract it to create both the upper and lower discriminant bands.

The third subsystem is the Results one, here we calculate the maximum angle of each routine, this defines the range of motion, to calculate the angle we create two vectors both start at shoulder, one ends at the hip and the other ends at the hand, with this two vectors we calculate the angle between them in any moment of the movement (shown in Figure 3), also we detect if there was unwanted movements of the patient when performing a certain kind of movement, this is estimated comparing the shoulder position with the other joints, this kind of natural movements are the compensation. There is another kind of result in which we compare the subject's curve of motion with the pre- estimated pattern of normal movement to indicate if there is an angle interval where the patient's move is out of the normal range, it calculates the percentage error of that interval and the exact angles of it. All the results include the regular videos, the depth streams and we generate a PDF report file with the results of each study. 


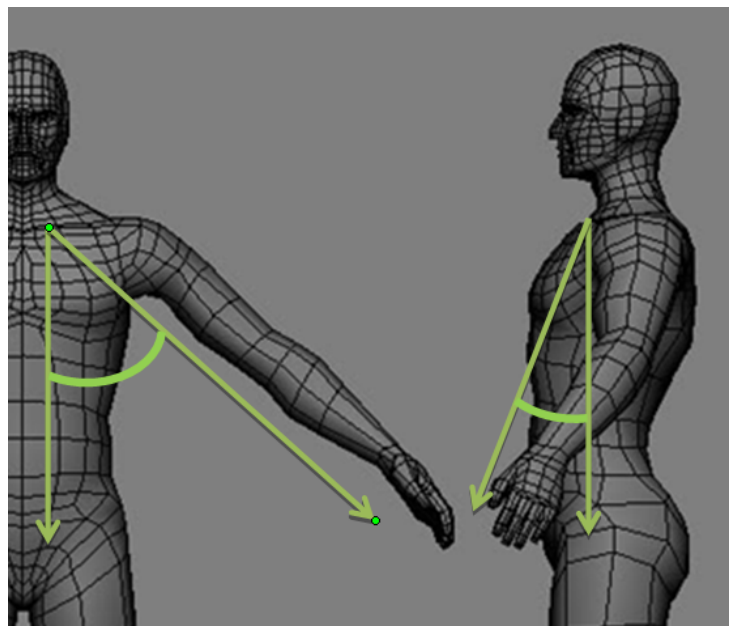

Fig. 3. Vector to calculate the range of motion

\section{Results}

We made captures among the local population with fifty three persons performing the five basic movement routines. They all share the same age range between 19 and 32 years old, the $84.90 \%$ were men and the remaining $15.01 \%$ were women. There was a wide range of weight from $47 \mathrm{~kg}$ to $160 \mathrm{~kg}$ with an average body mass index of 25.77 . The height was from $1.45 \mathrm{~m}$ to $1.98 \mathrm{~m}$. Some of the captured movements did not satisfy the needs to be included in the discriminant bands because of either incomplete movement routines or too much compensation, with the rest we created two discriminant bands for each of the movements. In Figure 4 and 5 we show the discriminant bands for the flection movement, the first one is for Y-Axis with only one hump and the second is for Z-Axis with two different humps. Normalizing the curves of movement was

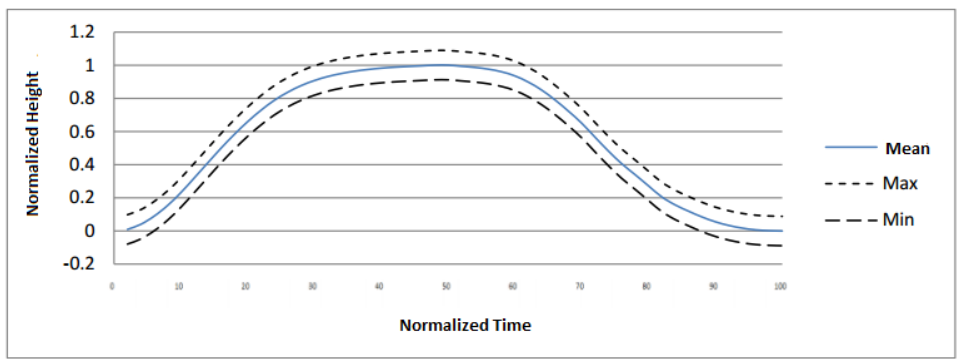

Fig. 4. Discriminant band for Y-Axis of Flection-Extension 


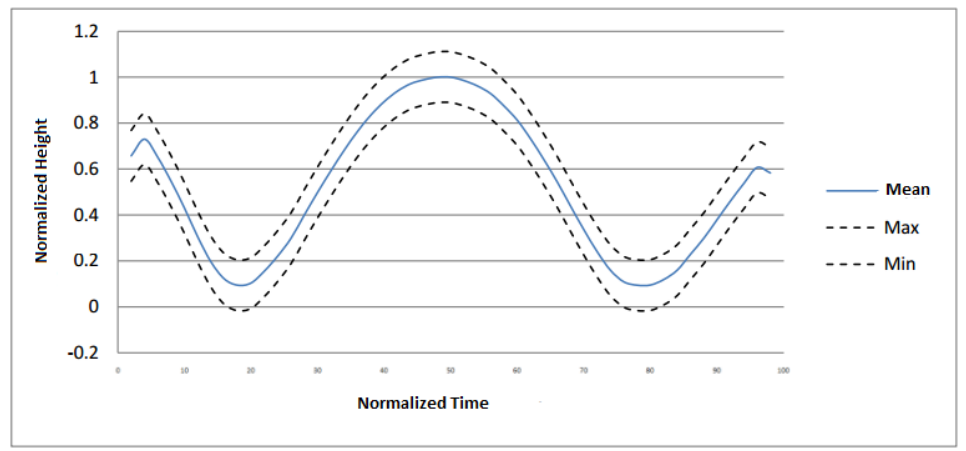

Fig. 5. Discriminant band for Z-Axis of Flection-Extension

a necessary task to do because it allowed us to compare people with different heights (in Figure 6 we see the comparison between the tallest and the smallest person of the group, having a difference of more than 50 centimeters, without the normalization we could not compare them, once that the two curves were normalized we can do comparison between them, see Figure 7), by this way we can compare different arms lenghts, weights, body mass index, disposition of motion depending of work or sport activity, different global times on the routine, different upward and downward time and different resting times. We generated

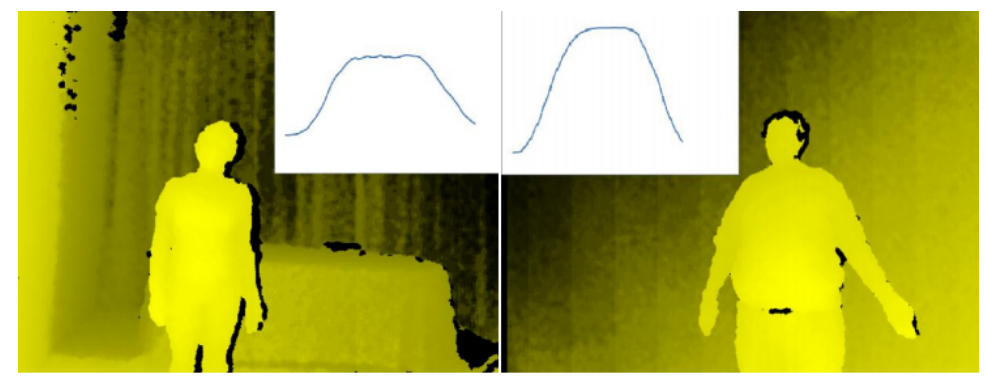

Fig. 6. Raw Motion curves of different sized people

results reports for different persons to validate the precision of arm lengths and height, the amount of error was around milimiters, it is important to mention that this precision is obtained when we have controlled illumination in the scene in other case the depth sensor has not this accuracy. We prove the results subsystem with regular data to see whether it fits to normal movement bands (Figure 8 ) and we also prove curves of movement out the regular pattern (FIgure 9), the result was possitive and they match with the expected medical analysis.

These previous results are done in inspections of active movements (movements made by ourselves), we also prove the system to work with passive inspections 


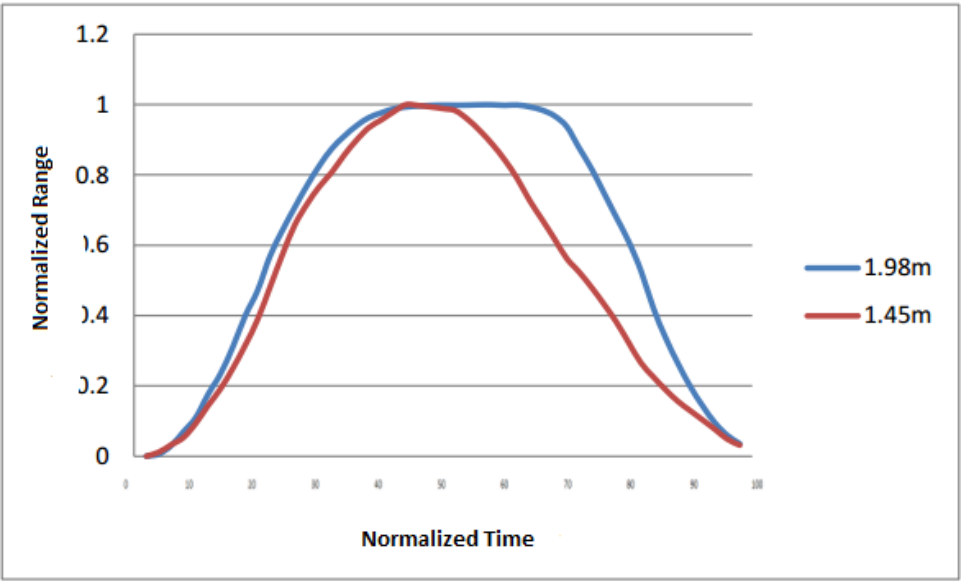

Fig. 7. Normalized Motion curves of different sized people

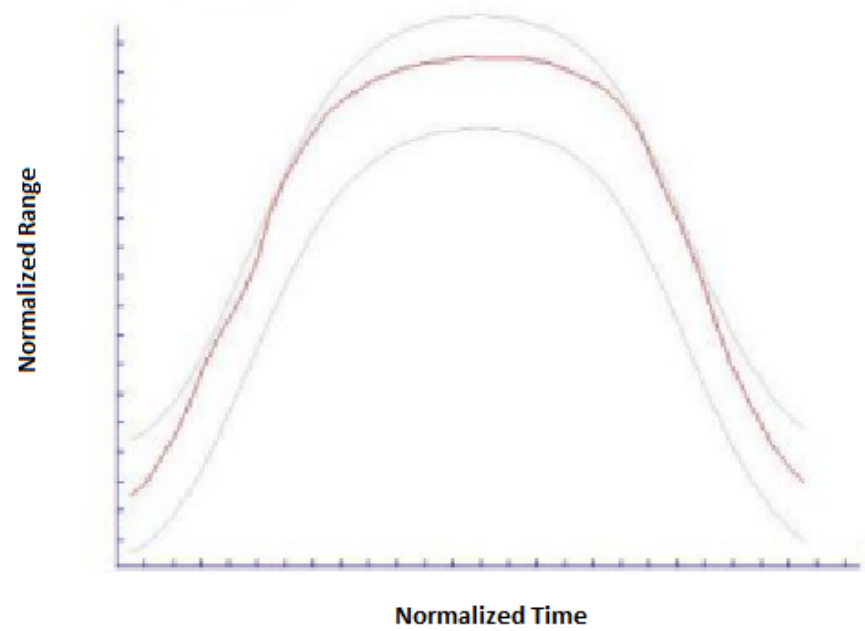

The whole movement was inside the normal range of movement, for Abduction and Adducition

Fig. 8. Motion curve that fits the Normal movement pattern 


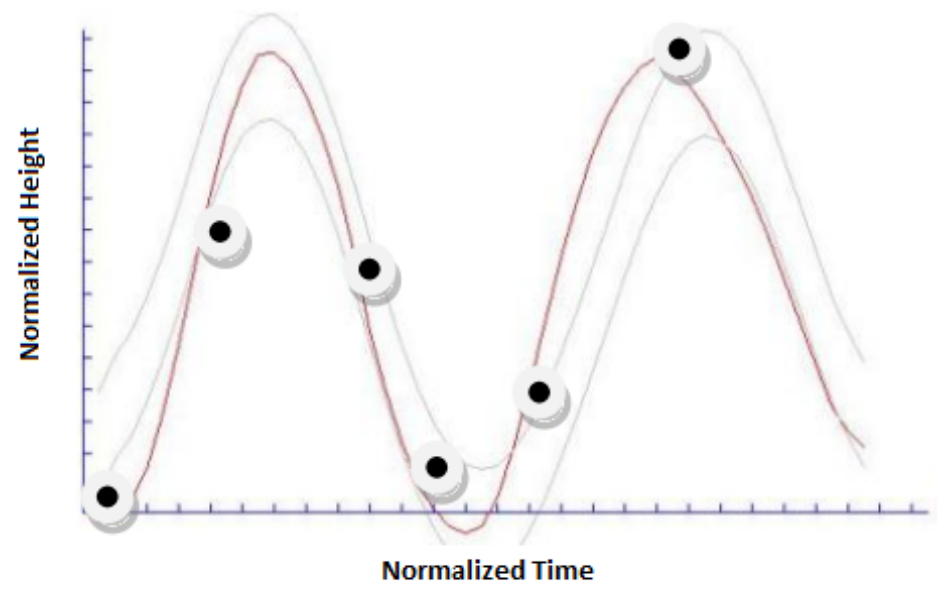

In Abduction, between $68.67^{\circ}-74.31^{\circ}$ there was an error of $3.77 \%$

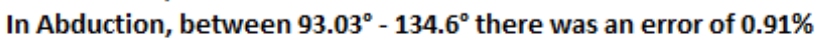
In Adduction, between $127.88^{\circ}-60.76^{\circ}$ there was an error of $1.33 \%$

Fig. 9. Motion curve that does not fit the Normal movement pattern

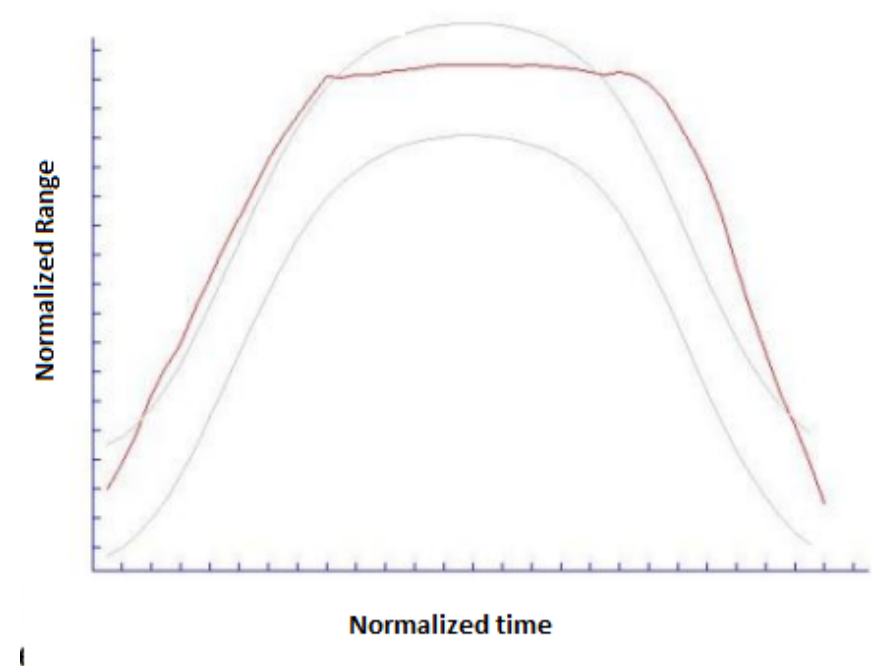

In Abduction, between $64.43-137.74^{\circ}$ there was an error of $2.72 \%$

Fig. 10. Motion curve of an inspection of movements against resistances (holding a weight) 
and movements against resistance. In the passive inspection we find possitive results but the physiotherapist has to be behind the patient, hidden from the depth sensor so the system do not detect the specialist or a part of him as an extension of the subject, if it is done right the system works perfectly. When working in movements against resistance we have better results because if the capture subject holds a weight or a stick (in kinesiotherapy are the most used objects in basic routines) the depth sensor does not recognized as an extension of the subject, so it does not modify the reading and recognition of the joints (Figure 10).

\section{Conclusion}

We show in this work, a system programmed with OpenSource tools, theoretically supported and validated by physiotherapy experts. The system allow us to capture patients with motor problems in a non-demanding, natural way, without the need to use heavy or unconfortable suits. It obtains numerical data, aided with mathematical and statistical processing, to deliver graphics and easy-tounderstand values that help when creating a diagnosis. The used tools allow obtaining results in real time, the stored information has a small weight to be stored, the assemblage of the cameras and the software installation are easy and fast. The devices used have a low cost compared with the commercial solutions, so in social security could be more reachable. We create local discriminant bands of normal movements, this is an achievement because there is not enough information of local range of motion or specific values, most of the information used was obtained from different countries not including Latin American people. As future work, the system can be adapted to study different joints of the body. The tool can be used in different hospitals and health centers, with all the gathered information we could create more accurate discriminant bands. Also with enough captures, we can create discriminant classes of specific characteristics, like age, gender or weight, that could help on a more focused study.

\section{References}

1. Freese, J.: Fitness terapeutico. Paidotribo, Barcelona, Espaa, (2006)

2. Xsens, www.xsens.com.

3. OpenNi, www.openni.org.

4. Middleware Nite v2.0, www.openni.org/file/nite.

5. Bouchrika, L. and Nixon, M.S.: Gait recognition by dynamic cues, Pattern Recognition ICPR 2008, pp 1-4, Tampa Florida, U.S., (2008)

6. Zaragoza University.: Research and Development in Ergonomics, http://i3a.unizar.es/en/content/idergo.

7. Andriacchi, T and Alexander, J.: Studies of human locomotion: past, present and Future, Deparments of Mechanical Engineering and Functional Restoration, Division of Biomechanical Engineering, Stanford University, (2000)

8. Menache, A.: Understanding motion capture for computer and videogames, Academic Press, San Diego, U.S. (2000)

9. Dyer, S.M.: Motion capture white paper, http://reality.sgi.com/employees/jamsb/mocap/. 